\title{
Application De La Télédétection Et Du Sig Au Suivi Des Formations Végétales De La Forêt Classée Des Trois Rivières Au Nord-Est Du Bénin
}

\author{
Soufouyane Zakari \\ Docteur, Laboratoire de Cartographie, Université d'Abomey-Calavi, Bénin \\ Ismaila Toko Imorou \\ Omer A. B. Thomas \\ Enseignants-Chercheurs, Maîtres de Conférences, Laboratoire de \\ Cartographie, Université d'Abomey-Calavi, Bénin

\section{Mama Djaouga} \\ Enseignant-Chercheur, Assistant Laboratoire de Cartographie, \\ Université d'Abomey-Calavi, Bénin

\section{Ousséni Arouna}

Enseignant-Chercheur, Maître-Assistant, Ecole des Sciences et Techniques du Bâtiment et de la Route, Université Nationale des Sciences, Technologies, Ingénierie et Mathématiques d'Abomey, Bénin

Doi: 10.19044/esj.2018.v14n15p450 URL:http://dx.doi.org/10.19044/esj.2018.v14n15p450

\begin{abstract}
The creation of protected areas was intended to counter the advance of the agricultural front and other forms of pressure on natural ecosystems, but today these pressures strongly affect these protected areas. The dynamics of the vegetation was studied in Trois Rivières forest reserve in north-eastern Benin, based on Remote Sensing and GIS techniques. This study aims to analyse the vegetation evolution and determine the drivers of that dynamics using 1995 Landsat TM, 2013 Landsat OLI-TIRS imageries and field surveys. The supervised classification based on the "Maximum likelihood" algorithm was derived from ENVI 5.0 software. The transition matrix derived from Intersect function of ArcToolbox in ArcGIS 9.3 software was used to calculate the different conversion of land cover. The transition matrix was used to measure the intensities and speeds of land cover categories changes from 'PontiusMatrix 22' 'and' 'Intensity Analysis02 programs. In addition, 200 household were surveyed from individual interviews and focus group on questionnaires and interview guides basis. The results show that the forest formations decreased from $48 \%$ in 1995 to $20.4 \%$ in 2013. The mosaics of fields and fallows have increased from $3 \%$ to $24 \%$. The intensity change per
\end{abstract}


land cover category shows that tree and shrub savannahs occurred the most significant change of $73 \%$ of the study area with a $34 \%$ of gain, $32 \%$ of stability and $7 \%$ of loss. In the order hand, the mosaics of fields and fallows, built up areas and dry dense forests occurred the biggest change. According to the perceptions of surveyed people, agriculture, logging and charcoal production are the main direct drivers of Trois Rivières forest reserve degradation.

Key Words: Remote Sensing, GIS, vegetation, Trois Rivières forest reserve, Benin.

\section{Résumé}

La création des aires protégées avait pour ambition de contrer l'avancée du front agricole et des autres formes de pressions sur les écosystèmes naturels, mais aujourd'hui ces pressions touchent fortement ces aires protégées. L'étude de la dynamique des formations végétales de la forêt classée des Trois Rivières au Nord-Est du Bénin à partir de la télédétection et du SIG vise à analyser l'évolution de la végétation et, de déterminer les facteurs de cette dynamique. Cette évolution a été analysée à partir des images satellites Landsat TM de 1995 et Landsat OLI-TIRS de 2013 combinées aux enquêtes de terrain. La classification supervisée basée sur l'algorithme "Maximum de vraisemblance"' a été réalisée avec le logiciel ENVI 5.0. Le logiciel ArcGIS 9.3 et les programmes "PontiusMatrix22', et "Intensity Analysis02', ont permis de mesurer les intensités et vitesses de changement des catégories d'occupation des terres. Par ailleurs, 200 personnes ont été interrogées à partir des entretiens individuels et des focus group. Les résultats révèlent qu'entre 1995 et 2013, les formations forestières sont passées de 48 $\%$ à 20, $4 \%$ et les mosaïques de champs et jachères de moins de $3 \%$ à $24 \%$. L'intensité de changement par unité d'occupation des terres montre que les savanes arborées et arbustives ont connu le plus important changement sur 73 $\%$ de la superficie du secteur d'étude avec $34 \%$ de gain, $32 \%$ de stabilité et $7 \%$ de perte. Par contre, les mosaïques de champs et jachères, les agglomérations et les forêts denses sèches ont connu les plus grandes vitesses de changement. De l'avis des enquêtés, l'agriculture, l'exploitation forestière et la carbonisation sont les principaux facteurs directs de dégradation de la forêt classée des Trois Rivières.

Mots Clés: Télédétection, SIG, végétation, Forêt classée des Trois Rivières, Bénin 


\section{Introduction}

La pression sur les ressources naturelles est particulièrement forte dans les pays pauvres où la pression démographique et la pauvreté font que les populations sont préoccupées par les objectifs de survie à court terme. L'Afrique tropicale est la plus durement touchée par les problèmes environnementaux.

Le Bénin n'est pas en marge de cette crise environnementale. En effet, les études sur les tendances actuelles de la dynamique de l'occupation des terres indiquent en général une régression du couvert végétal naturel au profit des terres agricoles et des pâturages (Thomas, 2014 ; FAO, 2010 ; Sinsin et Kampmann, 2010). Plusieurs études (Thomas, 2014 ; Houessou et al., 2013 ; Toko Mouhamadou et al., 2013 ; Kadiri, 2012 ; Arouna et al., 2011) sur la dynamique de formations végétales au Bénin ont montré que la tendance évolutive des écosystèmes forestiers est régressive. Par exemple, selon le scénario «environmental damage», le couvert forestier de la région de Doguè au centre du Bénin va disparaître d'ici à l'horizon 2025 (Orekan, 2007).

La création des aires protégées semblait être une panacée pour contrer l'avancée du front agricole et des autres formes de pressions sur les écosystèmes naturels. Malheureusement, plus de 60 ans après leur création, les forêts classées n'ont pas comblé toutes les attentes en matière de protection du couvert végétal (Toko et al., 2010). La forêt classée des Trois Rivières (FCTR), a été établie par l'arrêté N4524 SE du 06 septembre 1949 par l'administration coloniale (Hêkpazo, 1996). Elle a subi ces dernières années une dégradation de ses ressources naturelles. Ainsi, cette forêt classée est devenue un domaine privilégié où s'exercent les activités illicites telles que l'agriculture (champs de coton, de maïs et d'igname), la transhumance, le braconnage, l'exploitation forestière et la carbonisation. Face à ce constat, le suivi des formations végétales de la forêt classée de Trois Rivières paraît indispensable. Cela permettra in fine de renforcer la politique forestière pour une meilleure conservation des ressources phytogéniques.

Le cadre géographique de l'étude et la méthodologie adoptée pour analyser l'évolution de la végétation et ses facteurs explicatifs sont présentés dans la section suivante.

\section{Matériel et méthodes \\ Cadre d'étude}

La forêt classée des Trois Rivières est située dans les Communes de Bembèrèkè, de Kalalé, de Gogounou et de Ségbana au Nord-Est du Bénin entre $10^{\circ} 31^{\prime}$ 'et $10^{\circ} 45^{\prime}$ de latitude nord et entre $2^{\circ} 45^{\prime}$ ' et $2^{\circ} 35^{\prime}$ de longitude est (figure 1). Elle s'étend sur une superficie de $2685,3 \mathrm{Km}^{2}$ et est localisée dans la marge septentrionale de la zone soudano-guinéenne. La pluviosité est en moyenne de 1100 mm (ASECNA, 2013). La température moyenne annuelle 
est de $28^{\circ} \mathrm{C}$. L'harmattan et l'alizé maritime sont les deux types de vents dominants du secteur d'étude.

Les roches qui composent le substratum géologique du secteur d'étude sont constituées par les formations du socle granito-gneissique très ancien et des dépôts du quaternaire. Ce substratum géologiques est responsable de la mise en place de plusieurs types de formations pédologiques. On rencontre des sols ferrugineux tropicaux occupant la grande partie du cadre d'étude, des sols peu évolués, et des sols minéraux à gley. Le relief est très accidenté en raison de la pénéplaine faite de successions de croupes surbaissées caractéristiques des plaines soudaniennes (Faure et Volkoff, 1996 ; Dubroeucq, 1977). Le réseau hydrographique est constitué essentiellement de la rivière Sota et de ses affluents Tassiné et Bouri qui sont temporaires.

La FCTR est située dans le district phytogéographique du BorgouNord (Adomou et al., 2006). Les principaux types de formations végétales qu'elle renferme sont composés de galeries forestières, de forêts claires et savanes boisées, des savanes arborées et arbustives et des mosaïques de champs et de jachères.

Figure 1 : Localisation de la forêt classée des Trois Rivières au Bénin

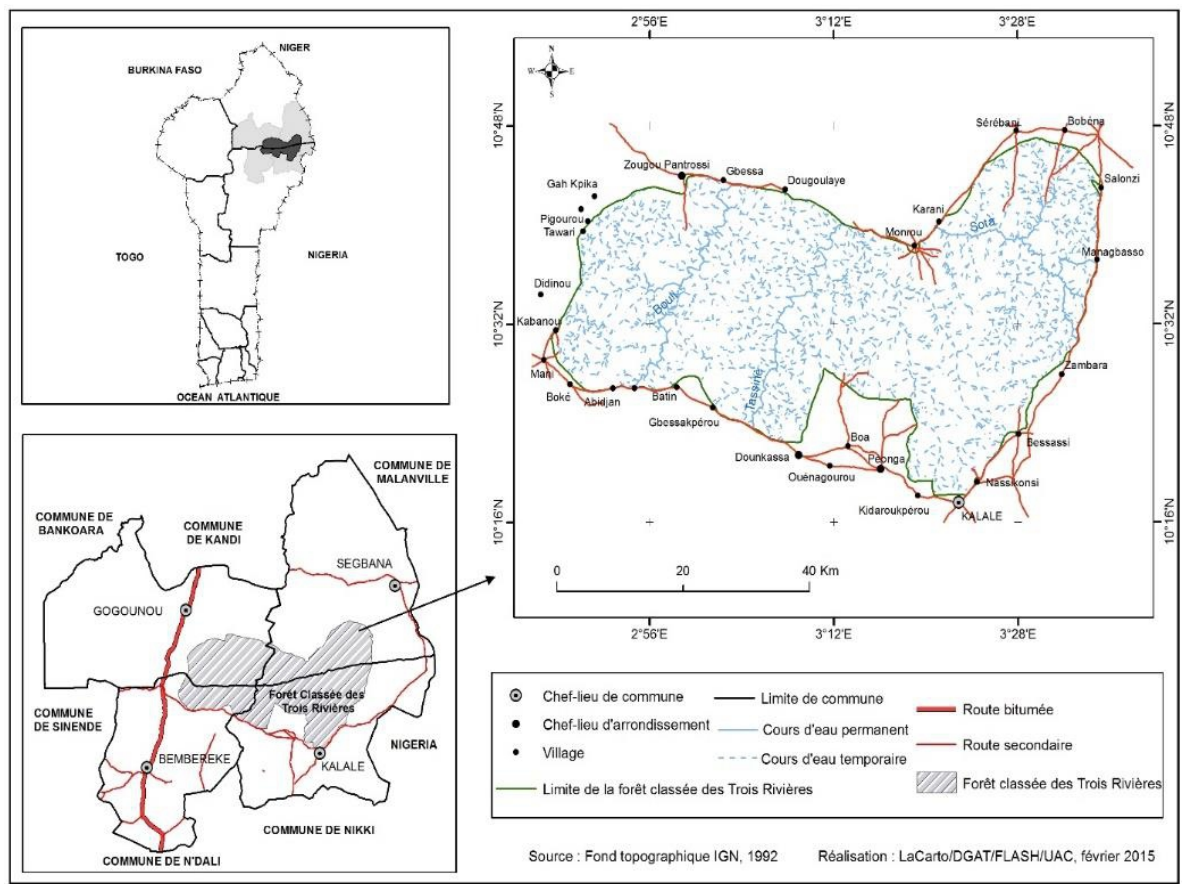

\section{Matériel utilisé}

\section{Données planimétriques}

Les données planimétriques utilisées au cours de cette étude sont : 
- $\quad$ carte topographique au 1/200 000 de l'Afrique de l'Ouest, feuille NC -31 XX Dunkassa ;

- $\quad$ images satellites TM de Landsat de 1995 du 25 novembre 1995 ; Paths : 191 et 192 ; Row : 53 ; résolution spatiale de $30 \mathrm{~m}$;

- $\quad$ images satellites OLI-TIRS de Landsat de 2013 du 12 février 2013 ;

Paths : 191 et 192 ; Row : 53 ; résolution spatiale de 30 m ;

- $\quad$ shapefiles des limites de la forêt classée des trois Rivières (FCTR).

Ces données ont permis de réaliser les cartes d'occupation des terres de la FCTR de 1995 et de 2013 avec le logiciel ArcGIS 10.1.

\section{Données socio-économiques}

Le matériel de collecte des données relatives aux déterminants de dégradation de la végétation est constitué de :

- questionnaire,

- guides d'entretien,

- grille d'observation.

\section{Méthodes utilisés}

Les méthodes concernes principalement les données planimétriques et les données socioéconomiques.

\section{Traitement des données planimétriques Interprétation des images satellites}

La classification supervisée par maximum de vraisemblance a été réalisée à l'aide du logiciel Envi 5.0. C'est une méthode de classification par pixel qui repose sur le postulat que la signature spectrale de chacun des pixels est représentative de la classe de végétation dans laquelle il se trouve. Huit (08) classes d'unités d'occupation des terres ont été retenues suivant la classification de l'IFN (2007). Le contrôle-terrain a été ensuite réalisé pour vérifier les classes de pixels issues de la classification. Sur le terrain, les coordonnées des formations et unités du paysage les plus illustratives ont été enrégistrées à l'aide du GPS. Au cours de cette phase, les pixels mis dans la classe de rejet ont été aussi identifiés.

\section{Exportation vers un Système d'Information Géographique}

Les fichiers de format raster des images interprétées ont été convertis en format vecteur dans le logiciel ArcGIS 9.3. Les cartes d'occupation des terres de 1995 et de 2013 de la FCTR ont été éditées en se basant sur les standards cartographiques. Les superficies des différentes formations végétales et des autres unités d'occupation des terres ont été calculées pour permettre la réalisation de la matrice de transition. 


\section{Matrice de transition entre 1995 et 2013}

La matrice de transition a permis de mettre en évidence les différentes formes de conversion qu'ont subies les formations végétales entre 1995 et 2013. Elle est constituée de $\mathrm{X}$ lignes et de Y colonnes. Le nombre de lignes de la matrice indique le nombre de classes d'occupation des terres au temps $\mathrm{t}_{0}$ (1995); le nombre Y de colonnes de la matrice est le nombre de classes d'occupation des terres converties au temps $t_{1}$ (2013) et la diagonale contient les superficies des formations végétales restées inchangées. Les transformations se font donc des lignes vers les colonnes. Les superficies de ces différentes classes d'occupation des terres ont été calculées à partir du croisement des cartes d'occupation des terres de 1995 et de 2013 à l'aide de la fonction Intersect de la boîte à outils ArcToolbox du logiciel ArcGIS 9.3.

\section{Taux de conversion}

Le taux de conversion d'une classe de végétation correspond au degré de transformation subie par cette classe de végétation vers d'autres classes. Il s'obtient à partir de la matrice de transition (Arouna, 2012) suivant la formule :

$\mathrm{TC}=\frac{\mathrm{ST}-\mathrm{Ss}}{\sum \mathrm{ST}} * 100$

Avec TC le taux de conversion, ST les superficies des unités d'occupation des terres issues de la conversion d'une formation végétale, Ss la superficie de la même formation restée stable à la date $t_{1}$.

\section{Intensités et vitesses des changements}

Les programmes d'analyse "PontiusMatrix22" et "Intensity Analysis02.xlms" (Aldwaik et Pontius, 2012) ont permis de mesurer (en \%) les intensités des changements selon les intervalles de temps, les unités d'occupation des terres et les transitions entre unités d'occupation des terres. Ce programme a été adapté à la matrice de transition de 1995-2013 du secteur d'étude pour générer deux graphiques montrant lesdites intensités au cours de cette période. Le premier graphique présente les intensités des changements survenus au sein de chaque catégorie. Par contre, le deuxième graphique montre les intensités de pertes et de gains par catégorie. A ces niveaux, l'état de rapidité des changements est déterminé grâce à la ligne verticale appelée ligne de zone uniforme. Si le graphe est à gauche de cette ligne, le changement est lent ou dormant ; par contre, si le graphe est à droite, le changement est rapide ou actif. 


\section{Collecte des données socio-économiques}

La collecte des données est axée sur l'utilisation des techniques de l'observation directe, des interviews semi-structurées et des interviews individuels structurés. La Méthode Accélérée de Recherche Participative (MARP) a été utilisée. Des entretiens individuels avec les différents acteurs et les personnes ressources et des focus group ont été réalisés dans les villages de Nassikonsi, Bessassi et Zambara (Commune de Kalalé) et dans les villages de Zougou Pantrossi, Gbessa et Dougoulaye (Commune de Gogounou). Quatre critères ont guidé le choix de ces villages : la proximité de la FCTR, l'accessibilité, les groupes socioprofessionnels (agriculteurs, agro-éleveurs, éleveurs, chasseurs, exploitants forestiers) et les groupes socioculturels (Batonnu, Peul et Boo).

Pour les groupes socioprofessionnels (éleveurs, chasseurs, exploitants forestiers) ayant un effectif limité, le choix a été raisonné. Par contre, le choix aléatoire est opéré pour les groupes socioprofessionnels (agriculteurs et agroéleveurs) majoritaires.

Les données statistiques sur l'effectif de ces différents acteurs n'étant pas disponibles, la taille de l'échantillon à enquêter au niveau de chaque catégorie d'acteurs est déterminée à partir de la formule suivante (Dagnelie, 1998) :

$$
n=\frac{4 \mathrm{P}(1-\mathrm{P})}{d^{2}}
$$

$\mathrm{P}$ étant la proportion de chaque catégorie d'acteurs obtenue à partir d'une enquête exploratoire, $d=10 \%$ (marge d'erreur variant de 0 à $20 \%$ ).

Au total, 200 acteurs ont été interrogés. Il s'agit des agriculteurs (100 individus), des agro-éleveurs (52 individus), des éleveurs (20 individus), des charbonniers (18 individus) et des exploitants forestiers (10 individus) riverains de la FCTR.

\section{Traitement des données socio-économiques}

\section{Valeur d'importance des déterminants de dégradation de la végétation}

La valeur d'importance des déterminants (IV) est la proportion d'enquêtés qui considèrent une activité ou un facteur comme un déterminant de dégradation de la végétation. Elle varie de 0 à 1 . Elle est déterminée par la formule suivante :

$$
\mathrm{IV}=\frac{\mathrm{n}_{\mathrm{is}}}{\mathrm{n}}
$$

Avec $\mathrm{n}_{\mathrm{is}}$ le nombre d'enquêtés qui considèrent une activité ou un facteur comme un déterminant de dégradation de la végétation et $\mathrm{n}$ le nombre total d'enquêtés. 


\section{Toile d'araignée ou roue de suivi évaluation}

La toile d'araignée ou le radar ou encore la roue de suivi évaluation a été utilisée pour faire une analyse comparative des déterminants selon les populations locales (Toko et al., 2010). Chaque paramètre a été représenté par un rayon gradué du centre vers la périphérie. L'échelle de graduation correspond aux valeurs d'importance des paramètres utilisés. Dans la présente étude, huit paramètres à savoir : l'agriculture, les changements climatiques, la carbonisation, la chasse, l'exploitation forestière, la récolte de miel, la transhumance et le non-respect des textes en vigueur par les différents acteurs ont été retenus. Cela a permis une représentation du radar à huit côtés. Cette démarche méthodologique adoptée à permis de d'atteindre les résultats présentés comme suit.

\section{Résultats}

\section{Cartographie de la dynamique des formations végétales de la FCTR}

En 1995, la forêt classée des Trois Rivières (FCTR) était dominée par les forêts claires/savanes boisées et les savanes arborées/arbustives. En 2013, la physionomie de la forêt est dominée par les arborées/arbustives et la mosaïque de champs et jachères. La figure 2 présente les cartes d'occupation des terres de la FCTR en 1995 et en 2013.

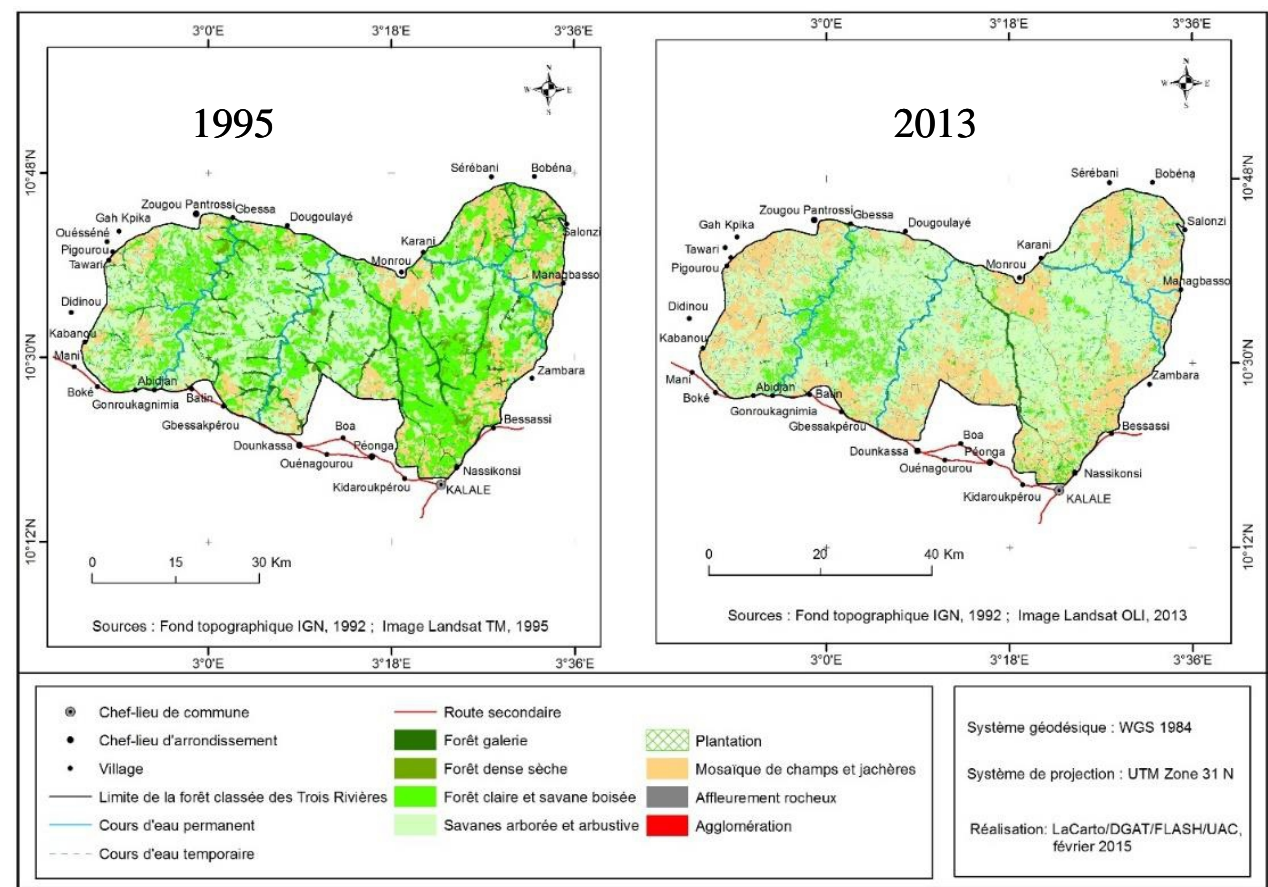

Figure 2 : Occupation des terres de la forêt classée des Trois Rivières entre 1995 et 2013

L'examen de la figure 2 permet de distinguer respectivement 8 et 7 classes d'occupation des terres en 1995 et 2013 dans la FCTR. La dynamique 
de l'occupation des terres de la FCTR entre 1995 et 2013 montre une forte anthropisation des écosystèmes naturels. La conversion des formations végétales et des autres unités ou catégories d'occupation des terres de la FCTR entre 1995 et 2013 est synthétisée sur le tableau 1.

Tableau 1 : Matrice de transition des formations végétales et autres catégories d'occupation des terres de 1995 à 2013

\begin{tabular}{ccccccccc}
\hline Unités de 1995 & FG & FCSB & SASA & PL & MCJ & AR & AG & Sup. total 1995 $\left(\mathrm{Km}^{2}\right)$ \\
\hline FG & 49,72 & 0 & 0 & 0 & 98,14 & 0 & 0 & 147,86 \\
FDS & 0 & 8,47 & 22,98 & 0 & 5,31 & 0 & 0 & 36,77 \\
FCSB & 0 & 142,99 & 686,76 & 0 & 142,81 & 0 & 0 & 972,56 \\
SASA & 0 & 106,77 & 907,69 & 0 & 87,87 & 0 & 0 & 1102,33 \\
PL & 0 & 0 & 0 & 12,31 & 0 & 0 & 0 & 12,31 \\
MCJ & 0 & 0 & 0 & 0,16 & 410,98 & 0 & 0,01 & 411,15 \\
AR & 0 & 0 & 0 & 0 & 0 & 2,03 & 0 & 2,03 \\
AG & 0 & 0 & 0 & 0 & 0 & 0 & 0,29 & 0,29 \\
\hline
\end{tabular}

\begin{tabular}{lccccccc}
\hline Sup. totale $2013\left(\mathrm{Km}^{2}\right)$ & 49,72 & 258,23 & 1617,43 & 12,47 & 745,11 & 2,03 & 0,30
\end{tabular} 2685,3

FG : Forêts galeries, FDS : Forêts denses Sèches, FCSB : Forêts Claires et Savanes Boisées, SASA : Savanes Arborées et Arbustives, PL : Plantations, MCJ : Mosaïques de Champs et de Jachères, AG : Agglomérations, Sup. totale : Superficie totale

Il ressort du tableau 1 que les superficies des formations végétales naturelles (forêts galeries, forêts denses sèches, forêts claires et savanes boisées) ont connu une nette régression avec plus de 2259,52 $\mathrm{Km}^{2}$ en 1995 à moins de $1925,38 \mathrm{Km}^{2}$ en 2013. Une partie importante de ces formations végétales s'est transformée en mosaïques de champs et de jachères qui occupent une superficie de $745,11 \mathrm{Km}^{2}$ de la FCTR en 2013. Il est important de signaler la convertion totale des forêts denses sèches en 2013 en d'autres catégories d'occupation que sont : les forêts claires et les savanes boisées, les savanes arborées et arbustives et les mosaïques de champs et jachères.

Le taux moyen de conversion est égal à $65,09 \%$; ce qui montre que plus de la moitié des formations végétales et des autres unités d'occupation des terres ont connu de transformation.

\section{Dynamique des forêts galeries}

De 1995 à 2013, la superficie des forêts galeries est passée 147,86 à $49,72 \mathrm{Km}^{2}$, soit une diminution de près de $80 \%$. Le taux moyen annuel de dégradation est de $6,97 \%$ avec un taux de conversion de $69,71 \%$. L'analyse de la matrice de transition montre que $98,14 \mathrm{Km}^{2}$ des forêts galeries ont été converties en mosaïque de champs et jachères et le reste $\left(49,72 \mathrm{Km}^{2}\right)$ est demeuré inchangé entre ces deux dates. Ce taux très élevé de conversion $(69,71 \%)$ des forêts galeries montre bien le degré de transformation de cette formation végétale. 


\section{Dynamique des forêts denses sèches}

Les forêts denses sèches se sont totalement transformées en d'autres unités entre 1995 et 2013. Ainsi, plus de la moitié des superficies des forêts denses sèches a été convertie en savanes arborées et arbustives (tableau 1). Par ailleurs, $5,31 \mathrm{Km}^{2}$ de forêts denses sèches ont été transformées en mosaïques de champs et jachères tandis que $8,47 \mathrm{Km}^{2}$ de forêts denses ont été converties en forêts claires et savanes boisées.

\section{Dynamique des forêts claires et savanes boisées}

De 1995 à 2013, la superficie des forêts claires et des savanes boisées est passée de $972,56 \mathrm{Km}^{2}$ à $258,23 \mathrm{Km}^{2}$ avec un taux de régression de 26,59 $\%$. L'analyse de la matrice de transition (tableau I) montre que plus du tiers des forêts claires et des savanes boisées $\left(686,76 \mathrm{Km}^{2}\right)$ a été transformé en savanes arborées et arbustives. D'autres portions de forêts claires et savanes boisées $\left(142,81 \mathrm{Km}^{2}\right)$ ont été transformées en mosaïques de champs et jachères. Le taux de conversion des forêts claires et des savanes boisées est de $85,30 \%$.

\section{Dynamique des savanes arborées et arbustives}

Le taux de conversion des savanes arborées et arbustives entre 1995 et 2013 est de 17,66 \%. L'examen de la matrice de transition (tableau 1) montre que plus de $87 \mathrm{Km}^{2}$ des savanes arborées et arbustives ont été converties en mosaïques de champs et jachères. Par ailleurs, 106,77 $\mathrm{Km}^{2}$ des savanes arborées et arbustives ont évolué vers les forêts claires et savanes boisées. Par contre, près de $700 \mathrm{Km}^{2}$ de forêts claires et savanes boisées ont été convertis en savanes arborées et arbustives.

Intensité de changement par catégorie d'occupation des terres entre 1995 et 2013

La figure 3 présente l'intensité de changement par catégorie d'occupation des terres entre 1995 et 2013. 


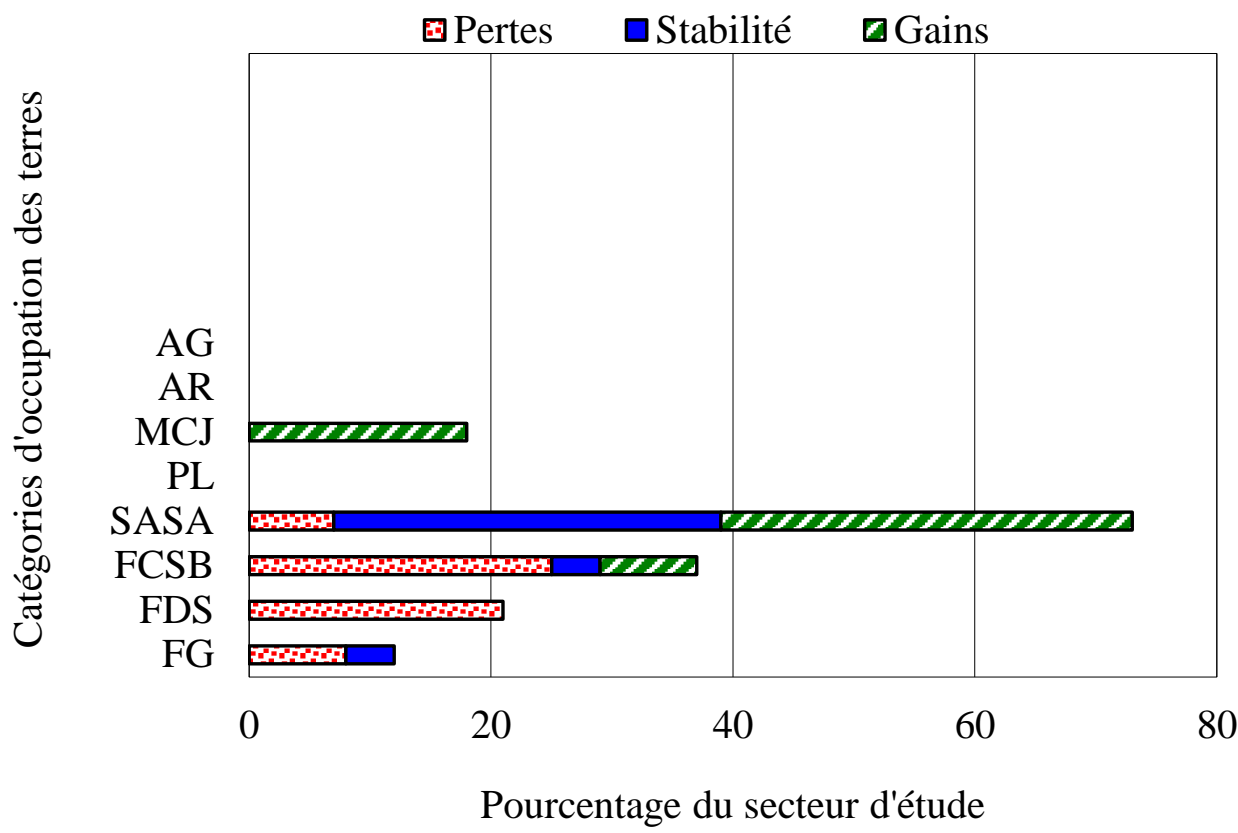

Figure 3: Intensité de changement par catégorie d'occupation des terres entre 1995 et 2013 FG : Forêt galerie ; FDS : Forêt dense sèche ; FCSB : Forêt claire et savane boisée ; SASA : Savanes arborée et arbustive ; SS : Savane saxicole ; MCJ : Mosaïque de champs et jachères ; PL : Plantation ; AR : Affleurement rocheux ; AG : Agglomération.

L'examen de la figure 3 révèle que toutes les catégories d'occupation des terres ont connu de changement de 1995 à 2013. Ainsi, les savanes arborées et arbustives ont connu le plus important changement sur $73 \%$ de la superficie du secteur d'étude avec $34 \%$ de gain, $32 \%$ de stabilité et $7 \%$ de perte. Elles sont suivies des forêts claires et savanes boisées $(37 \%$ de la superficie du secteur d'étude) avec $8 \%$ de gain, contre $4 \%$ de stabilité et 25 $\%$ de perte. Par ailleurs, les forêts denses sèches ont connu exclusivement de perte sur $20 \%$ de la superficie du secteur d'étude. Par contre, les mosaïques de cultures et jachères ont connu de gain sur $18 \%$. Enfin, les forêts galeries ont connu $8 \%$ de perte et $4 \%$ de stabilité.

\section{Intensité et vitesse de changement des catégories d'occupation des terres entre 1995 et 2013}

La figure 4 présente l'intensité et la vitesse de changement des catégories d'occupation des terres entre 1995 et 2013. 


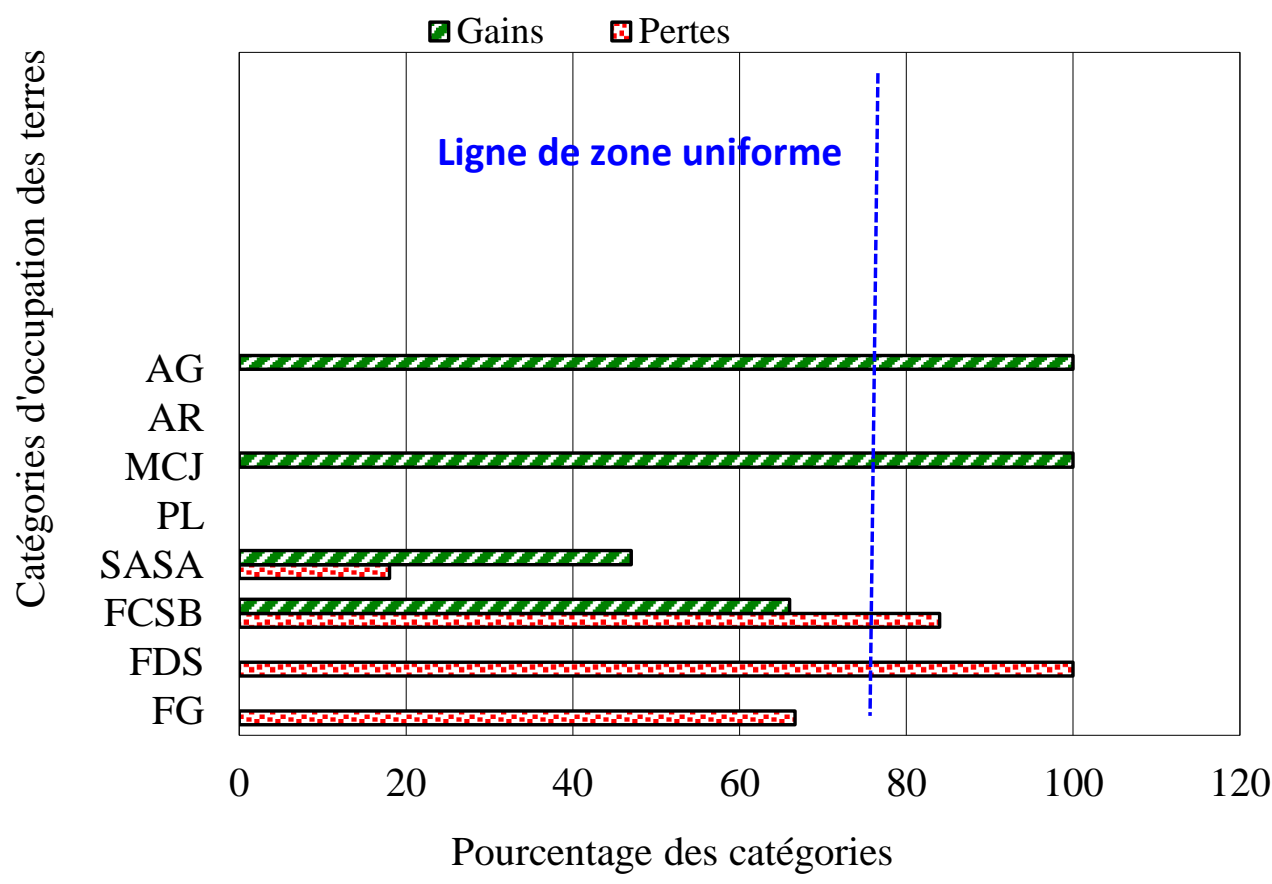

Figure 4: Intensité et vitesse de changement des catégories d'occupation des terres entre 1995 et 2013

FG : Forêt galerie ; FDS : Forêt dense sèche ; FCSB : Forêt claire et savane boisée ; SASA : Savanes arborée et arbustive ; SS : Savane saxicole ; MCJ : Mosaïque de champs et jachères ; PL : Plantation ; AR : Affleurement rocheux ; AG : Agglomération.

De l'analyse de la figure 4, il ressort que les changements opérés au niveau des différentes catégories d'occupation des terres ont été rapides en dehors des affleurements rocheux et des plantations qui sont restées stables. Cependant, la vitesse de changement varie suivant les formations végétales. En effet, ce sont les unités d'occupation des terres, composées respectivement de mosaïques de champs et jachères et d'agglomérations d'une part et des forêts denses sèches d'autre part qui ont connues les plus grandes vitesses de changement sur $100 \%$ du secteur d'étude, respectivement en terme de gain et de perte. Elles sont suivies des forêts claires et savanes boisées (65\% de gain et $85 \%$ de perte); des forêts galeries ( $65 \%$ de perte) puis des savanes arborées et arbustives (50\% de gain et $18 \%$ de perte).

\section{Perceptions locales des déterminants de dégradation de la végétation}

L'agriculture, la transhumance, la carbonisation et l'exploitation forestière constituent les principaux déterminants de dégradation de la végétation de la FCTR selon les populations locales (figure 5). 


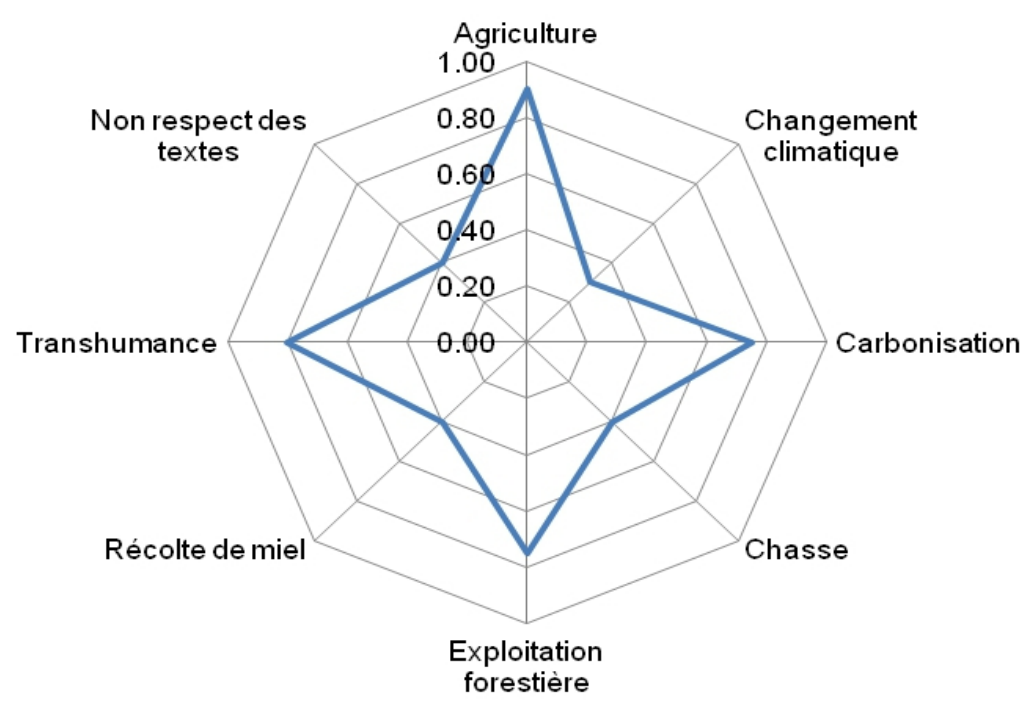

Figure 5 : Perceptions locales des déterminants de dégradation de la végétation

L'examen de la figure 5 révèle que l'agriculture $(0,85)$, la transhumance $(0,80)$, la carbonisation $(0,77)$ et l'exploitation forestière $(0,75)$ sont les principaux déterminants de dégradation de la végétation de la FCTR.

L'agriculture à travers les défrichements culturaux sur brulis, convertit complètement les formations végétales en espaces agricoles. La disponibilité de fourrage et de points d'eau dans la FCTR fait de cette forêt classée, une aire d'accueil des troupeaux transhumants en provenance du Niger, du Nigéria et du Burkina-Faso. L'afflux de ces troupeaux transhumants fait que la capacité de charge de la FCTR est vite atteinte. Les bouviers se livrent à l'émondage et à l'étêtage des ligneux fourragers. Par ailleurs, la carbonisation et l'exploitation forestière transforment les formations végétales relativement denses en formations végétales ouvertes à travers les trouées créées par ces activités.

Quant à la chasse et la récolte de miel, elles sont perçues par les populations locales comme des déterminants indirects de dégradation de la végétation car les chasseurs en cherchant à rendre accessibles les aires giboyeuses, et les récolteurs de miel en cherchant à renvoyer ou tuer les abeilles, provoquent les feux de végétation tardifs qui consument la biomasse végétale. Certains récolteurs de miel abattent systématiquement les espèces ligneuses mellifères afin d'accéder à la ruche. Le non-respect des textes en vigueur par les différents acteurs est un déterminant indirect de la dégradation de la végétation selon les populations. Enfin, les changements climatiques qui se manifestent, entre autres par l'assèchement du milieu naturel et la faible 
pluviosité sont aussi perçus par les populations comme les déterminants naturels de dégradation des formations végétales naturelles.

\section{Discussion}

\section{Dynamique des formations végétales}

Les formations végétales de la forêt classée des Trois Rivières (FCTR) connaissent une évolution essentiellement régressive. Cette régression est beaucoup plus importante dans les formations fermées comme les forêts galeries, les forêts denses sèches et les forêts claires. Par contre, les mosaïques de cultures et jachères et les savanes arborées et arbustives ont connu une extension de leurs superficies. La forte savanisation de cette forêt classée qui, auparavant était dominée par des formations végétales fermées telles que les forêts denses sèches et les forêts claires est essentiellement liée aux activités anthropiques. Or dans les conditions normales, les écosystèmes de cette forêt devraient évoluer vers la végétation climacique qu'est la forêt dense sèche. Malheureusement, cette végétation climacique n'existe plus dans le secteur d'étude. Plusieurs auteurs (Moussa, 2014 ; Karimou, 2013 ; Toko Imorou, 2013 a et b ; Arouna et al., 2010 ; Toko et al., 2010 a et b) ont fait le même constat dans la région soudanienne et dans la zone de transition soudanoguinéenne du Bénin. Il en a été de même que Ballo et al.., 2016 dans le Terroir de Ziguéna, zone cotonnière de au Mali.

\section{Approches Télédétection et SIG dans la cartographie des changements spatio-temporels}

L'apport de la télédétection dans le suivi du couvert végétal est de plus en plus grandissant surtout avec l'affinement progressif de la résolution spatiale des images satellites qui est passée de $80 \mathrm{~m}$ en 1972 pour Landsat MSS à moins de $1 \mathrm{~m}$ pour les images Quickbird et Pléiades de nos jours. De ce fait, les études sur la déforestation dans la zone intertropicale se sont multipliées (Toko Mouhamadou, 2014). Ces études proposent avec une très grande précision des diagnostics ainsi que des analyses diachroniques pour expliquer l'évolution de la déforestation (Bamba et al., 2008 ; Dibi N'Da et al., 2008 ; Tsayem et al., 2001).

Les résultats de la présente étude montrent la pertinence des différentes méthodes utilisées pour l'étude de la dynamique des formations végétales. Ainsi, ces résultats montrent l'évolution de chaque formation végétale, une donnée essentielle pour l'élaboration et la mise en œuvre d'un plan d'aménagement forestier. Ceux-ci permettront aussi de prendre des mesures de reconstitution des formations végétales dégradées. Ces résultats confirment ceux des travaux de plusieurs chercheurs qui ont démontré que l'utilisation de la Télédétection, des SIG et des données géospatiales est indispensable pour la cartographie de la végétation et de l'occupation des terres (Toko 
Mouhamadou, 2014 ; Maman et al., 2011 ; Hountondji, 2008 ; Orekan, 2007 ; Achard et al., 1996). Selon ces auteurs, les images satellites jouent un rôle incontournable dans le processus de cartographie et d'aménagement des écosystèmes forestiers. C'est ce qui explique, par exemple, que de nombreuses études portant sur l'analyse de la dynamique des écosystèmes des aires protégées (Parc National du W du Niger, forêts classées de Bellefoungou, de Ouénou-Bénou, de l'Alibori Supérieur et des Monts Kouffé) ont utilisé des techniques de Télédétection et des SIG (Toko Imorou, 2013a ; Toko et al., 2010a ; Clerici et al., 2007; Barbier et al., 2006 ; Couteron et Kokou, 1992).

\section{Déterminants de la dégradation des formations végétales}

La dynamique régressive des formations végétales de la FCTR est surtout imputable à l'agriculture, à l'exploitation forestière, à la carbonisation et à la transhumance. Par ailleurs, la chasse, la récolte de miel et le non-respect des textes en vigueur par les différents acteurs sont les déterminants indirects de cette dégradation. Plusieurs auteurs (Karimou, 2013 ; Oloukoï et al., 2013 ; Toko Imorou, 2013 a et b; Arouna et al., 2011 ; Toko et al., 2010 a et b; Sounon Bouko et al., 2007 ; Orekan, 2007 et Arouna, 2002) ont fait le même constat selon lequel l'agriculture est la principale cause de régression du couvert végétal. Selon Toko Imorou (2013 a) et Toko et al. (2010 a), l'agriculture pratiquée autour des forêts classées de l'Alibori Supérieur et de Ouénou-Bénou situées dans la même zone agro-écologique que la forêt classée des Trois Rivières est dominée par la culture du coton et de l'igname qui sont des plantes héliophiles. Le défrichement anarchique, l'abattage systématique des essences même celles qui ont des valeurs socioéconomiques et la culture sur brûlis ont entraîné le recul du couvert végétal. Ainsi, le paysage qui était auparavant composé de formations boisées s'est progressivement transformé en une mosaïque de champs et de jachères. Toutes ces observations rejoignent celles effectuées dans la présente étude dans la forêt classée des Trois Rivières.

Les résultats de Moussa (2014), Toko Imorou (2013 a), Kadiri (2012) et Toko et al. (2010 a) sur l'impact de l'exploitation forestière dans les forêts classées de Bellefoungou, de Ouénou-Bénou et de l'Alibori Supérieur sont similaires à ceux obtenus dans la FCTR où cette activité a entraîné des trouées caractérisées par l'absence des espèces ligneuses. Dans le secteur d'étude, l'exploitation forestière a entraîné la raréfaction des espèces telles que Khaya senegalensis, Afzelia africana et Pterocarpus erinaceus. De ce fait, ces exploitants se sont rués sur des espèces comme Anogeissus leiocarpa, Daniellia oliveri, Isoberlinia doka et Isoberlinia tomentosa dont la densité des individus exploitables est de plus en plus faible. Pour Akoegninou et Akpagana (1997), la raréfaction des grands arbres tels que Afzelia africana, Anogeissus leiocarpa, Burkea africana, Pterocarpus erinaceus et Isoberlinia doka dans les forêts classées est due aux activités anthropiques. 
Les transhumants, les braconniers et les récolteurs de miel sont le plus souvent responsables des mutilations des essences fourragères et mellifères et des feux de végétation dans la FCTR pour favoriser la repousse des graminées, ou dégager les milieux giboyeux ou renvoyer les abeilles. Ces feux de végétation et mutilations contribuent à la régression du couvert végétal. Plusieurs auteurs sont parvenus à la même conclusion selon laquelle, la structure de la végétation est sous la dépendance des activités anthropiques telles que le régime des feux de végétation et le surpâturage (Gould et al., 2006; Devineau et Fournier, 2005 ; Thornes, 2005 ; Mappin et al., 2003 ; Houinato et al., 2001 ; Hansen et al., 2000).

La carbonisation qui était une activité méconnue des populations riveraines de la FCTR a de nos jours pris une ampleur importante à cause de la forte demande des consommateurs et la raréfaction du bois de chauffe. Les sources d'approvisionnement étant de plus en plus rares, les populations se rabattent dans les forêts classées où la végétation ligneuse est encore importante. Le même constat est fait dans les forêts classées de Bellefoungou, de Ouénou-Bénou et de l'Alibori Supérieur par Moussa (2014), Toko Imorou (2013 a), Kadiri (2012) et Toko et al. (2010 a). La carbonisation participe à la déstructuration de la végétation. Il en est de même de l'abattage systématique des espèces mellifères par les récolteurs de miel pour accéder aux ruches. Toutes ces activités nuisent dangereusement à la structure de la végétation par les trouées qu'elles créent. Cette exploitation abusive est en partie favorisée par le laxisme de l'administration forestière et le non-respect des textes en vigueur par les autorités à divers niveaux.

\section{Conclusion}

L'utilisation des techniques de Télédétection et du SIG a joué un rôle incontournable dans le suivi de la dynamique de l'occupation des terres de la forêt classée des Trois Rivières. La cartographie diachronique a montré la dégradation des formations végétales naturelles et l'évolution des espaces agricoles et des plantations. A partir de ces résultats, l'élaboration et la mise en œuvre d'un plan d'aménagement forestier pourront être faites et les mesures de reconstitution des formations végétales dégradées pourront être prises.

\section{References:}

1. Achard F., Ansseau C., Beaubien J., Defourny P., Jaton A., Lemieux G., Royer A. \& Thomson K. (1996) : La télédétection des forêts. In: AUPELF et al. (Editor). Précis de Télédétection Volume II. AUF, Québec, pp. 227-265.

2. Adomou A.C., Sinsin B. \& van der Maesen L. J. G. (2013): Phytosociological and chorological approaches to phytogeography: a meso-scale study in Benin. Syst. Geogr. pl. 76: 155-178 
3. Akoegninou A. \& Akpagana K. (1997) : Etude cartographique et dynamique de la végétation de l'aire classée de la colline de Savalou (Bénin). J. Bot. Soc. Bot. Fr., 3 : 69-81.

4. Aldwaik S.Z. \& Pontius Jr. R. G. (2012) : Intensity analysis to unify measurements of size and stationarity of land change by interval, category and transition. Landscape and urban planning, 106: 103-114

5. Arouna O. (2012): Cartographie et modélisation prédictive des changements spatio-temporels de la végétation dans la commune de Djidja au Bénin: implications pour l'aménagement du territoire. Thèse de doctorat FLASH/UAC, $246 \mathrm{p}$.

6. Arouna O., Toko I., Djogbenou C. P. \& Sinsin B. (2011): Comparative analysis of local populations' perceptions of socio-economic determinants of vegetation degradation in sudano-guinean area in Benin (West Africa). 2011. International Journal of Biodiversity and Conservation, 3(7): 327-337.

7. Arouna O., Toko Imorou I., Djogbenou C. P. \& Sinsin B. (2010): Impact de la dynamique spatio-temporelle de l'occupation du sol sur la végétation en zone soudano-guinéenne au Bénin. Rev. Sc. Env. Univ., Lomé (Togo), nº06 : 161-186.

8. Arouna O. (2002) : L'exploitation des ressources biologiques et la dynamique de la forêt classée de l'Alibori Supérieur au Bénin (secteur de l'arrondissement de Bagou). Mémoire de Maîtrise de Géographie, DGAT/FLASH/UAC/Bénin, 125 p.

9. ASECNA (2013) : Agence pour la Sécurité de la Navigation Aérienne en Afrique et à Madagascar. Station météorologique de Kandi, Bénin.

10. Ballo A., Traore S.S., Coulibaly B., Diakité C.H., Diawara M., Traore A., Dembélé S. (2016): Pressions anthropiques et dynamique d'occupation des Terres dans le Terroir de Ziguéna, zone cotonnière du Mali. European Scientific Journal February edition vol.12, No.5 ISSN: 1857 - 7881 (Print) e - ISSN 1857- 7431.

11. Bamba I., Mama A., Neuba D.F.R., Koffi K.J., Traoré D., Visser M., Sinsin B., Lejoly J. \& Bogaert J. 2008: Influence des actions anthropiques sur la dynamique spatio-temporelle de l'occupation $\mathrm{du}$ sol dans la province du Bas-Congo (R.D. Congo). Science \&Nature Vol. $5 \mathrm{~N}^{\circ} 1: 49-60$.

12. Barbier N., Couteron P., Lejoly J., Deblauwe V. et Lejeune O. (2006) : Self-organised vegetation patterning as fingerprint of climate and human impact on semiarid ecosystems. J. Ecol. 94: 537- 47.

13. Clerici N., Bodini A., Eva H., Grégoire J. M., Dulieu D. \& Paolini C. (2007): Increased Isolation of Two Biosphere Reserves and Surrounding Protected Areas (WAP: W-Arly-Pendjari, Ecological Complex, West Africa). J Nat Conserv, 15 : 26-40. 
14. Couteron P. \& Kokou K. (1992): Parc National du W (Niger) : typologie et cartographie de la Végétation du Parc National et de la réserve de faune de Tamou. Montpellier : Engref-Unesco. Collet C. (1992): Systèmes d'information géographique en mode image. Lausanne, Presses polytechniques et universitaires romandes, Col. Gérer l'environnement, $\mathrm{n}^{\circ} 7,186 \mathrm{p}$.

15. Devineau J-L. \& Fournier A. (2005): To what extent can simple plant biological traits account for the response of the herbaceous layer to environmental changes in fallow-savanna vegetation (West Burkina Faso, West Africa) ? Flora, 200 : 361-375.

16. Dibi N'Da H., Kouakou N'Geussan E., Egnankou Wadja M. \& Affian K. (2008) : Apport de la télédétection au suivi de la déforestation dans le Parc National de la Marahoué (Côte d'Ivoire), Revue Télédétection, pp.17-34.

17. Dubroeucq D. (1977) : Notice explicative de la carte pédologique de reconnaissance de la république populaire du Bénin. Feuille de Parakou. ORSTOM, Paris, 37 p.

18. FAO (2010): http://web.icppgr.fao.org, consulté le 23-05-10.

19. Faure P. \& Volkoff B. (1996): Différenciation régionale des couvertures pédologiques et litho-géomorphologie sur socle granitogneissique du Bénin (Afrique occidentale). C. R. Acad. Sci., 322 : 393400.

20. Gould W.A., Gonzalez G. \& Carrero Rivera G. (2006) : Structure and composition of vegetation along an elevatioal gradient in Puerto Rico. Journal of Vegetation Science, 17: 653-664.

21. Hansen A. J., Rotella J. J., Kraska M. P. V. \& Brown D. (2000) : Spatial patterns of primary productivity in the Greater Yellowstone Ecosystem Landscape. Ecology, 15: 505-522.

22. Hêkpazo P. (1996): Recueil des textes de classement et de déclassement des forêts, des périmètres de reboisement et des réserves en République du Bénin. PGRN/MDR/Cotonou, Bénin, 102 p.

23. Hountondji Y. C. H. (2008) : Dynamique environnementale en zones sahélienne et soudanienne de l'Afrique de l'Ouest : Analyse des modifications et évaluation de la dégradation du couvert végétal. Thèse de doctorat, Département des Sciences et Gestion de l'Environnement Faculté des Sciences. Université de Liège, Liège, Belgique. 131 p.

24. Houéssou L. G., Tèka O., Toko I., Lykke A. M. \& Sinsin B. (2013): Land Use and Land-Cover Change at "W" Biosphere Reserve and Its Surroundings Areas in Benin Republic (West Africa). Environment and Natural Resources Research, 3(2): 87-101. 
25. Houinato, M., Sinsin, B. \& Lejoly, J. (2001): Impact des feux de brousse sur la dynamique des communautés végétales dans la forêt de Bassila (Bénin). Acta Bot. Gallica, 148(3) : 237-251.

26. Kadiri A. B. (2012) : Etude phytoécologique de la forêt classée de Ouénou Bénou (Commune de Bembèrèkè). Mémoire de Maîtrise de Géographie, DGAT/FLASH/UAC, 93 p.

27. Karimou S. (2013): Dynamique phytogéographique de la marge septentrionale de la zone soudano-guinéenne dans la Commune de Bembèrèkè : secteur centre (Arrondissement d'Ina). Mémoire de Maîtrise de Géographie, DGAT/FLASH/UAC, 122 p.

28. Maman M. I., Mahamane A., Mbow C., Saadou M. \& Bachmann Y. (2011) : Dynamique spatio-temporelle des forêts claires dans le Parc national du W du Niger (Afrique de l'Ouest). Sécheresse, 22 : 108-16.

29. Mappin K. A., Pate J. S. \& Bell T. L. (2003) : Productivity and water relations of burnt and long-unburnt semi-arid shrubland in Western Australia. Plant and Soil, 257 : 321-340.

30. Moussa A. D. (2014): Cartographie des changements spatiotemporels de l'occupation du sol dans la forêt classée de Bellefoungou (Commune de Djougou). Mémoire de Maîtrise de Géographie, DGAT/FLASH/UAC, $82 \mathrm{p}$.

31. Oloukoï J., Mama V.J. \& Agbo F. B. (2013) : Modélisation de la dynamique de l'occupation des terres dans le Département des Collines au Bénin. Télédétection 6 (4): 305-323.

32. Orekan V. O. A. (2007): Implementation of the local land-use and land-cover change model CLUE-s for Central Benin by using socioeconomic and remote sensing data. Dissertation, University of Bonn, $230 \mathrm{p}$.

33. Scouvart M. \& Lambin E. F. (2013) : Approche systémique des causes de la déforestation en Amazonie brésilienne : syndromes, synergies et rétroactions. Environnement, $3: 241-254$.

34. Sinsin B. (1998) : Etude de la faune et de son habitat dans les Forêts Classées des Trois Rivières, de Ouénou-Bénou et de l'Alibori Supérieur Rapport de mission. Ambassade Royale des Pays-Bas, Cotonou, Bénin, 47 pages.

35. Sinsin B. \& Kampmann D. (eds). (2010) : Atlas de la Biodiversité de l'Afrique de l'Ouest, Tome I : Bénin. Cotonou \& Frankfurt/Main, 726 p.

36. Sounon Bouko B., Sinsin B. \& Goura Soulé B. (2007): Effets de la dynamique d'occupation du sol sur la structure et la diversité floristique des forêts claires et savanes au Bénin. Tropicultura, 25(4): 221-227.

37. Thomas O. (2014) : Cartographie de l'occupation et utilisation du sol 
dans la périphérie Sud de la forêt classée des trois rivières au Bénin. Science et technique, Lettres, Sciences sociales et humaines, 30(2): 177-189.

38. Thornes J. B. (2005) : Coupling erosion, vegetation and grazing. Land Degrad. Develop., 16: 127-138.

39. Toko Imorou I. (2013 a) : Dynamique des formations végétales de la forêt classée de Ouénou-Bénou au Nord-Bénin. BenGéo., 13 : 18-35.

40. Toko Imorou I. (2013 b) : Impact des activités anthropiques sur la végétation en région soudanienne : La Commune de Kandi au Bénin. J. Rech. Sci. Univ. Lomé (Togo), 2013, Série B, 15(3) : 65-77.

41. Toko I., Arouna O. \& Sinsin B. $(2010$ a) : Cartographie des changements spatio-temporels de l'occupation du sol de la forêt classée de l'Alibori Supérieur au Nord-Bénin. 2010. BenGéo., 7 : 2239

42. Toko I., Orthmann B., Porembski S. \& Sinsin B. (2010 b) : Facteurs déterminant la répartition spatiale de la flore et de la structure de la végétation dans les sous bassins d'Aguimo et de Térou-Wannou au centre du Bénin. Rev. Sc. Env. Univ., Lomé (Togo), 006 : 5-24.

43. Toko Mouhamadou I. (2014): Facteurs déterminants de la fragmentation des écosystèmes forestiers : cas des îlots de forêts denses sèches de la forêt classée des Monts Kouffé et de sa périphérie au Bénin. Thèse de Doctorat, EDP/FLASH/UAC ; Abomey-Calavi, Bénin, $221 \mathrm{p}$.

44. Toko Mouhamadou I., Toko Imorou I., Gbègbo M. C. \& Sinsin B. (2013) : Structure et composition floristiques des forêts denses sèches de la région des Monts Kouffé au Bénin. Journal of Applied Biosciences, 64: 4787 - 4796.

45. Tsayem-Demaze M., Polidori L. \& Fotsing J. M., 2001. Caractérisation multi-échelle et multi-capteur de la déforestation tropicale amazonienne. Bulletin de la Société Française de Photogrammétrie et de Télédétection, 161 : 74-84. 